\title{
Prediction of fetal lung immaturity using gestational age, patient characteristics and fetal lung maturity tests: a probabilistic approach
}

\author{
L. D. E. Wijnberger $\cdot$ M. de Kleine $\cdot$ H. A. M. Voorbij • \\ B. Arabin - H. W. Bruinse - G. H. A. Visser • \\ P. M. M. Bossuyt $\cdot$ B. W. J. Mol
}

Received: 25 December 2008 / Accepted: 2 March 2009 / Published online: 19 April 2009

(c) The Author(s) 2009. This article is published with open access at Springerlink.com

\begin{abstract}
Objectives The lecithin/sphingomyelin (L/S) ratio and the lamellar body count (LBC) can be used to predict respiratory distress syndrome (RDS).

Design We performed a retrospective cohort study among consecutive women who underwent amniotic fluid sampling for the assessment of fetal lung maturity. Logistic regression was used to construct models for the prediction of RDS in three gestational age categories, with models
\end{abstract}

L. D. E. Wijnberger $(\bowtie) \cdot$ M. de Kleine $\cdot$ H. W. Bruinse

G. H. A. Visser - B. W. J. Mol

Department of Obstetrics, Neonatology and Gynaecology, University Medical Center Utrecht,

KJ02.507.0 Wilhelmina Children's Hospital,

PO Box 85090, 3508 AB Utrecht, The Netherlands

e-mail: 1.wijnberger@xs4all.nl

B. W. J. Mol

e-mail: b.w.mol@amc.nl

L. D. E. Wijnberger

Department of Obstetrics,

Leiden University Medical Center, Leiden, The Netherlands

H. A. M. Voorbij

Departments of Clinical Chemistry,

University Medical Center Utrecht, Utrecht, The Netherlands

B. Arabin

Departments of Obstetrics, Isala Clinics,

Location Sophia, Zwolle, The Netherlands

P. M. M. Bossuyt

Department of Clinical Epidemiology and Biostatistics,

Academic Medical Center, Amsterdam, The Netherlands

B. W. J. Mol

Department of Obstetrics and Gynaecology,

Academic Medical Center, Amsterdam, The Netherlands based on clinical characteristics only, clinical characteristics and the LBC, and on clinical characteristics and $\mathrm{L} / \mathrm{S}$ ratio.

Results When amniotic fluid was collected $<30$ weeks, the specificity of the LBC was $30 \%$ and the sensitivity $100 \%$. Addition of the L/S ratio increased the specifity to $60 \%$, for a sensitivity of $100 \%$. When amniocentesis was performed between 30 and 33 weeks, addition of the L/S ratio only marginally improved the performance of the LBC.

Conclusions At a gestational age $<30$ weeks, the L/S ratio has additional value over the LBC. Above 30 weeks of gestation, single use of the LBC seems sufficient.

Keywords Fetal lung maturity - Lamellar body count . $\mathrm{L} / \mathrm{S}$ ratio $\cdot$ Respiratory distress sydrome (RDS)

\section{Introduction}

The lecithin/sphingomyelin (L/S) ratio and the lamellar body count (LBC) are two invasive tests used to assess fetal lung maturity in pregnancies at risk for preterm delivery [1-3]. Many studies have reported on the accuracy of these tests. In a recent meta-analysis in which we summarized six studies in which both tests were compared, the LBC was found to be at least as accurate as the L/S ratio [4]. However, accuracy is not the only issue that is of importance in establishing their value for clinical practice. In this context, Richardson and Heffner [5] recently stated that although tests for fetal lung maturity were mature, their interpretation was not. They hypothesized that due to the high risk of lung immaturity at low gestation, testing for fetal lung maturity was not useful before a gestational age of 32-33 weeks. On the other hand, they assumed the prevalence of RDS and other complications after a gestational 
age of 37 weeks to be low, thus implicating that testing was also not useful near term. They suggested that there is a need for studies in which pre-test probabilities, test performance and post-test probabilities are related. The aim of the present study was therefore to evaluate the contribution of $\mathrm{L} / \mathrm{S}$ ratio and $\mathrm{LBC}$ in the prediction of fetal lung immaturity. To do so, we constructed prediction models using patient characteristics and then evaluated whether the prediction changed after adding results from L/S ratio and LBC.

\section{Methods}

\section{Patients}

The study was performed in two large teaching hospitals with a tertiary referral function for perinatal medicine in The Netherlands. We reviewed in retrospect the medical files of consecutive women who underwent amniotic fluid sampling for the assessment of fetal lung maturity. Amniotic fluid was collected by transabdominal amniocentesis. Exclusion criteria were an uncertain gestational age, fetal anomalies which could possibly interfere with the occurrence of RDS, and monoamniotic twin pregnancies. Women with ruptured membranes in whom amniotic fluid was collected vaginally, were also excluded from the study. When repeated sampling was performed in one woman, only the last sample before delivery was used for analysis. For each patient, we recorded the reason for admission, use of antenatal glucocorticoids and tocolytic drugs, ultrasound and Doppler findings, multiple pregnancy, gestational age at time of testing and at delivery, contamination of the samples with blood or meconium and neonatal outcome. Gestational age was calculated from the first day of the last menstrual period, or from first trimester ultrasonography. All samples were obtained for clinical purposes. Clinical management of pregnancies was based on the maternal and/ or fetal condition and on the outcome of the fetal lung maturity tests. The occurrence of RDS was diagnosed according to clinical symptoms of respiratory stress and findings on chest radiographs [6]. Clinical symptoms of respiratory stress included the need for continuous positive airway pressure for at least $24 \mathrm{~h}$. Moreover, an experienced neonatologist, who was unaware of the outcome of the fetal lung maturity tests, reviewed all chest radiographs of the infants with reported RDS. Women of whom the outcome of the infant was unknown or of whom the infant died within $24 \mathrm{~h}$ after delivery without developing RDS, were excluded from the analysis.

Multiple regression analysis indicated that both the L/S ratio and the LBC varied statistically significant with gestational age. Both were statistically different in the patients who delivered more than 14 days after amniocentesis (both $P$ values $<0.01)$. However, the $\mathrm{L} / \mathrm{S}$ ratio and the $\mathrm{LBC}$ differed not statistically significant between women who delivered within $48 \mathrm{~h}$ after amniocentesis, women that delivered between $48 \mathrm{~h}$ and 7 days after amniocentesis, and women who delivered between 7 and 14 days after amniocentesis [7].

In view of these data, we excluded women who delivered more than 14 days after the amniocentesis. The exclusion of these women introduces a bias called verification bias, since women with low fetal lung maturity test results were more likely to be excluded than women with normal or high fetal lung maturity test results [8]. We controlled for verification bias by calculating for each women the probability of verification, i.e. the probability that a woman delivered within 2 weeks after amniotic fluid collection [9]. This probability was calculated with a logistic regression model, in which the occurrence of verification was the dependent variable, and gestational age and the results of fetal lung parameters were the independent variables [10]. This probability was used as a weight factor in the statistical calculations that are described later in the "Methods" section.

\section{Laboratory methods}

Immediately after arrival at the laboratory, amniotic fluid samples were centrifuged for $5 \mathrm{~min}$ at $450 \mathrm{~g}$. In one center the LBC was performed on a Technicon $\mathrm{H}^{*} 1$ haematology analyser (Bayer Diagnostics, Tarrytown, USA) during the first 3 years and on a Cell-Dyn 4000 (Abbott Diagnostics, Santa Clara, USA) from 1999. The other center used only the Cell Dyn 1600 (Abbott Diagnostics, Santa Clara USA). Measurements on the Technicon $\mathrm{H}^{*} 1$ were made with the laser cell counter (helium neon laser at $632.8 \mathrm{~nm}$ ) with measurement on the basis of low angle scatter signal with fixed thresholds for counting between $2 \mu$ and $30 \mu \mathrm{m}$ (platelet channel). Measurements on the Cell-Dyn were made with the aperture impedance cell counter in the erythrocyte/platelet channel. The histogram of the platelets was used for the LBC. Both methods were calibrated against each other. In both centers the L/S ratio was determined by thin-layer chromatography according to Gluck et al. [1] and performed in duplicate. The variation between the measurements was about $15 \%$ for both the $\mathrm{L} / \mathrm{S}$ ratio and the LBC. We tested several amniotic fluid samples in both centers to verify comparability of $\mathrm{L} / \mathrm{S}$ and $\mathrm{LBC}$ results and did not find statistically significant differences.

\section{Statistics}

We distinguished three categories of women based on the gestational age at amniocentesis: (1) <30 weeks; (2) 
between 30 and 33 weeks; (3) >33 weeks. In each category, we used stepwise logistic regression to construct a model for the prediction of RDS, using clinical characteristics, but not the results of L/S ratio and LBC. Clinical characteristics considered were reason for admission, use of antenatal glucocorticoids and tocolytic drugs, multiple pregnancy, ultrasound findings, diabetes type I, gestational diabetes, gender of the fetus, and gestational age at time of amniocentesis. The probability of verification was used as relative weight in this logistic model. Selection of variables is usually performed with a significance level of 0.05 . However, the incorrect exclusion of a prognostic factor would be more deleterious than including too many factors [9]. Therefore, multivariable analysis included all variables with a $P$ value $<0.30$. We then added the LBC to the model in the three gestational age groups. Subsequently, we plotted the probability of RDS as predicted by the model without the LBC against the probability of RDS predicted by the model with the LBC added to the clinical characteristics. Similarly, the $\mathrm{L} / \mathrm{S}$ ratio was forced into the models with the $\mathrm{LBC}$, and we then plotted the probability predicted with the model with the LBC added to the clinical characteristics against the probability predicted with $\mathrm{L} / \mathrm{S}$ ratio added to the clinical characteristics.

\section{Results}

During the study period, 363 women met the inclusion criteria. From these women, 13 infants died within $24 \mathrm{~h}$ without developing RDS and from 16 infants we had an incomplete follow up because of transport to another hospital. There were 154 women who delivered within $48 \mathrm{~h}$ after amniocentesis, 82 who delivered within $48 \mathrm{~h}$ and 7 days, 49 who delivered within 7 and 14 days, and 49 who delivered more than 14 days after amniocentesis. For the analysis, we excluded the 49 women who delivered more than 14 days after amniocentesis. Thus, 285 women were available for analysis, of whom 61 infants (21\%) developed RDS. In 31 women (11\%) the $\mathrm{L} / \mathrm{S}$ ratio and in 19 women (7\%) the LBC was not determined.

There were 26 mother with diabetes type I (7.9\%) and 32 mothers with gestational diabetes $(9.7 \%)$. Tocolysis was applied in 44 women (14\%), whereas 167 women (9.7\%) had had steroids antenatally. There were 152 male babies (46\%), 85 (26\%) were born from a multiple pregnancy and 69 were below the 5 th percentile $(21 \%)$. The logistic models that were constructed to predict the occurrence of RDS are shown in Table 1. Diabetes of the mother [OR 3.3, 95\% confidence interval (CI) $0.58-18, P$ value 0.18 ], antenatal administration of glucorticosteroids (OR 0.97, 95\% CI $0.29-3.3, P$ value 0.96 ), use of tocolysis (OR $0.31,95 \%$ CI $0.06-1.5, P$ value 0.14$)$, being born from a multiple preg-
Table 1 The three logistic models that were used to calculate the probability of RDS

\begin{tabular}{llll}
\hline & $\begin{array}{l}\text { Model without } \\
\text { fetal lung } \\
\text { maturity tests }\end{array}$ & $\begin{array}{l}\text { Model } \\
\text { with LBC }\end{array}$ & $\begin{array}{l}\text { Model with } \\
\text { L/S ratio }\end{array}$ \\
\hline Beta & 19.7321 & 22.2879 & 12.3899 \\
Gestational & -0.0951 & -0.1024 & -0.0502 \\
$\quad$ age (per day) & & 0.0369 & 0.0326 \\
Fetal growth $(\%)$ & 0.0245 & -0.0752 & - \\
LBC $(1,000 / \mu \mathrm{L})$ & - & - & -1.2332 \\
L/S ratio & - & & \\
\hline
\end{tabular}

The probability can be calculated by the formula $P=\exp (\beta) /$ $(1+\exp (\beta))$. The $\beta$ can be calculated from the patient characteristics; for example for a woman with a gestational age of 210 days and an estimated fetal growth on the $\mathrm{p} 25$ in which $\beta$ is calculated as $19.7321-$ $210 \times 0.0951+25 \times 0.0245$

nancy (OR $0.37,95 \%$ CI $0.11-1.2, P$ value 0.105 ), and being male (OR $0.74,95 \%$ CI $0.24-2.3, P$ value 0.60 ), whereas fetal growth as well as the L/S ratio and the LBC were verified (Table 1).

There were 50 women who underwent amniocentesis before 30 weeks, and 41 of them delivered within 14 days after amniocentesis, i.e. fetal lung maturity status could be verified. From these women 25 infants developed RDS $(25 / 42=61 \%)$. Figure 1a shows the probability of RDS as predicted without fetal lung maturity tests in relation to the probability of RDS that was calculated after taking into account the result of the LBC. This Figure shows that without performing fetal lung maturity tests, it is not possible to distinguish between infants who will develop RDS and infant who will not develop RDS. After taking into account the results of the LBC, two out of 16 women whose infant did not develop RDS could have been identified as being at low risk, i.e. below $10 \%$. Figure $1 \mathrm{~b}$ shows the result of adding the $\mathrm{L} / \mathrm{S}$ ratio to the model with the LBC. Another five women whose infant did not develop RDS could be identified as being at low risk, thus increasing the specificity to over $60 \%$, for a sensitivity of $100 \%$.

There were 96 women with amniotic fluid collection between 30 and 33 weeks and 76 delivered within 14 days after the procedure. From these women, 25 infants developed RDS $(25 / 76=33 \%)$. Figure 2a shows the probability of RDS as predicted without fetal lung maturity tests in relation to the probability of RDS that was calculated after taking into account the results of the LBC. Without performing fetal lung maturity tests, it appeared not to be possible to distinguish between women whose infant will develop RDS and whose infant will not develop RDS (Fig. 2a). However, after taking into account the result of the LBC, 20 out of 51 women whose infant did not develop RDS could have been identified as being at low risk, at the 
Fig. 1 Prediction model for fetal lung immaturity at gestational age $<30$ weeks. a Probability of RDS, without taking into account fetal lung maturity tests, as compared to the probability calculated after taking into account the result of the LBC. b Probability of RDS, taking into account only LBC and after addition of the L/S ratio
Fig. 2 Prediction model for fetal lung immaturity at gestational age 30-33 weeks. a Probability of RDS, without taking into account fetal lung maturity tests, as compared to the probability calculated after taking into account the result of the LBC. b Probability of RDS, taking into account only LBC and after addition of the $\mathrm{L} / \mathrm{S}$ ratio
Gestational age $<30$ weeks
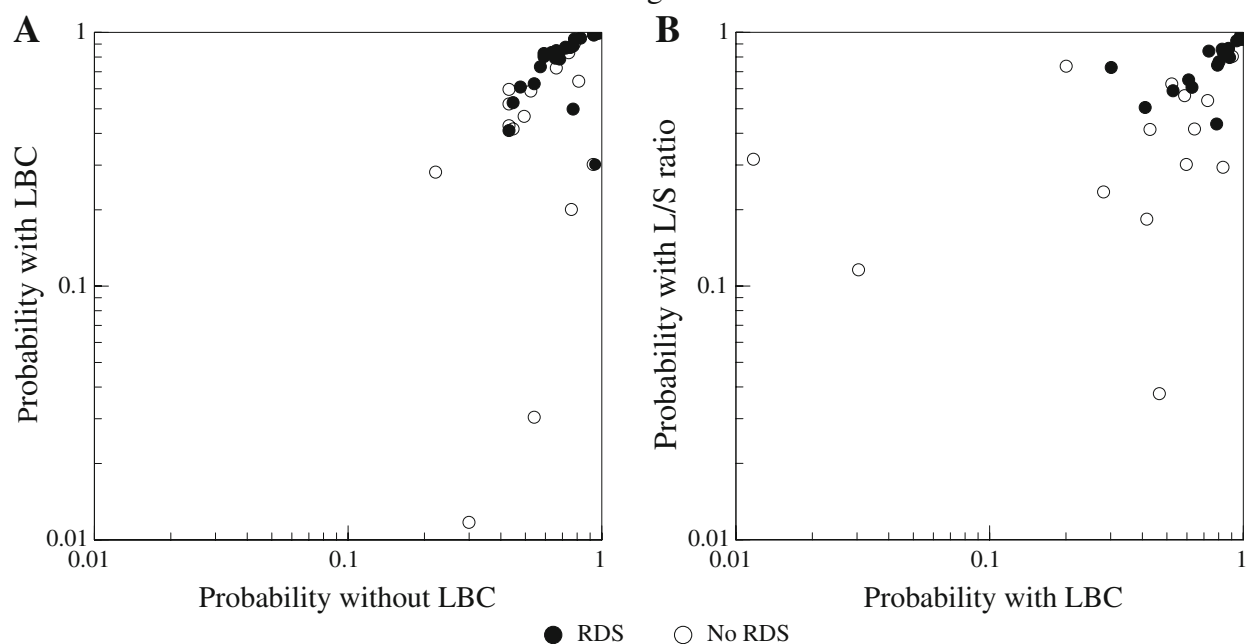

Gestational age 30-33 weeks

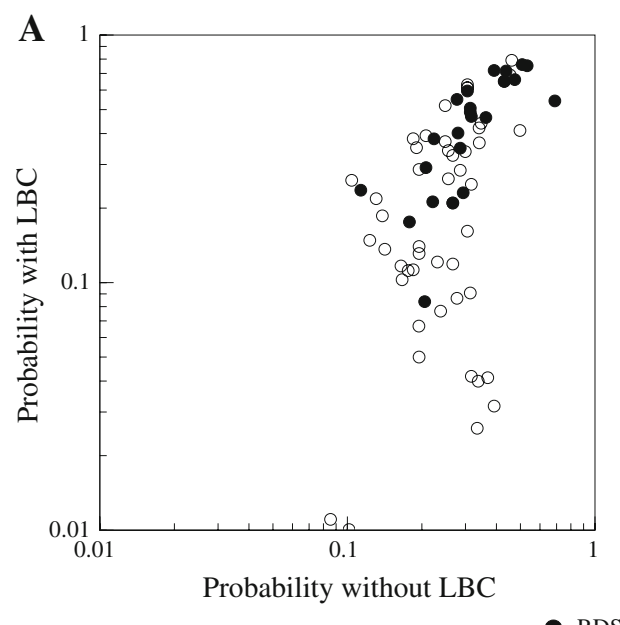

cost of one false-negative test result. Figure $2 b$ shows the result of adding the $\mathrm{L} / \mathrm{S}$ ratio to the model with the $\mathrm{LBC}$. Only another three women whose infant did not develop RDS could be identified as being at low risk, whereas the false-negative test result according to the LBC was identified correctly as being at high risk due to a low L/S ratio.

There were 184 women who had an amniocentesis after 33 weeks and 167 of them delivered within 14 days after the procedure. From these women 10 infants developed $\operatorname{RDS}(10 / 167=6 \%)$. Figure 3 a shows the probability of RDS as predicted without fetal lung maturity tests in relation to the probability of RDS that was calculated after taking into account the results of the LBC. Without fetal lung maturity tests, about 25 of the 157 women whose infant did not develop RDS could be identified as being at low risk. After taking into account the LBC, another 25 women whose infant did not develop RDS could have been identified as being at low risk, without false-negative test results. Figure $3 b$ shows the result of adding the L/S ratio to the model with the LBC. There were only 38 women in whom the L/S ratio was performed. Addition of the results of the $\mathrm{L} / \mathrm{S}$ ratio to the model resulted only in one out of 13 cases in which the probability of RDS changed from more than $10 \%$ to a probability of lower than $10 \%$.

\section{Discussion}

This study reports on the contribution of $\mathrm{L} / \mathrm{S}$ ratio and $\mathrm{LBC}$ to the prediction of fetal lung immaturity. To do so, we constructed prediction models using patient characteristics, and then evaluated whether the prediction changed after adding results from $\mathrm{L} / \mathrm{S}$ ratio and $\mathrm{LBC}$. In women who had an amniocentesis below 30 weeks, the specificity of the LBC was $30 \%$ for a $100 \%$ sensitivity. Use of the $\mathrm{L} / \mathrm{S}$ ratio increased the specificity to $60 \%$, for a sensitivity of $100 \%$. In women who had amniocentesis between 30 and 33 weeks, the specificity of the LBC was $40 \%$ for a sensi- 
Fig. 3 Prediction model for fetal lung immaturity at gestational age $>33$ weeks. a Probability of RDS, without taking into account fetal lung maturity tests, as compared to the probability calculated after taking into account the result of the LBC. b Probability of RDS, taking into account only LBC and after addition of the $\mathrm{L} / \mathrm{S}$ ratio

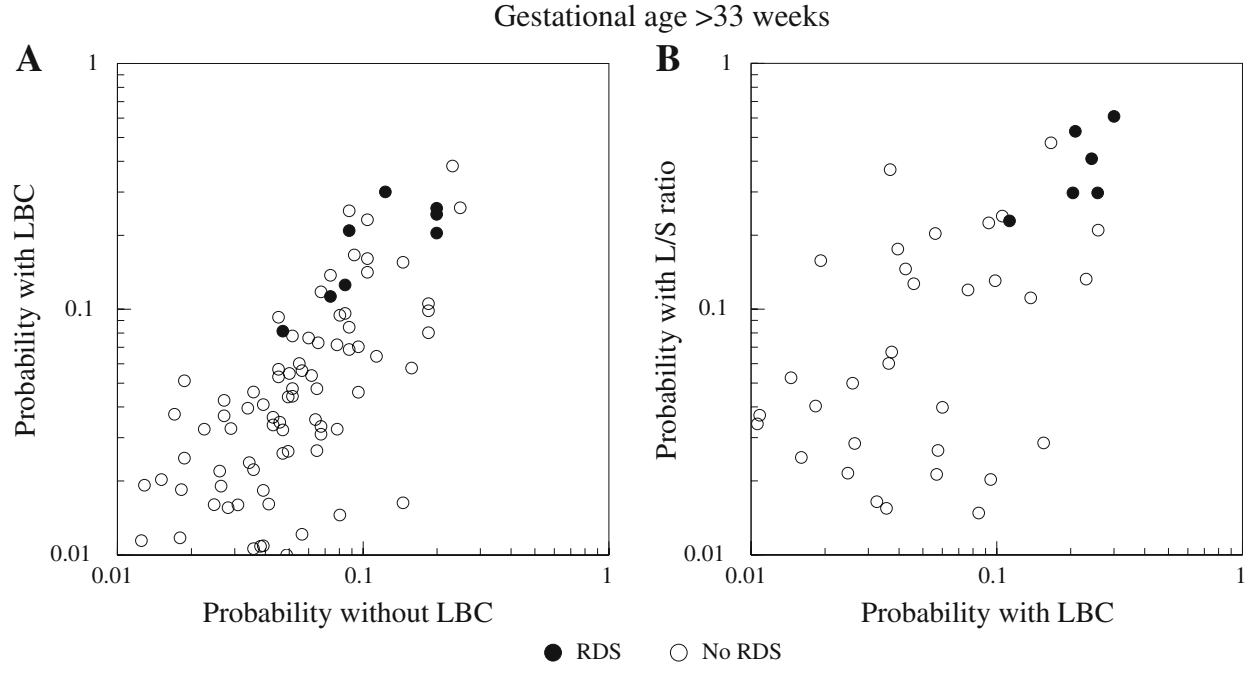

tivity of $96 \%$. Addition of the $\mathrm{L} / \mathrm{S}$ ratio improved the specificity only slightly. In women who had amniocentesis above 33 weeks, $15 \%$ could have been identified as being at low risk before amniocentesis. Addition of the LBC increased the specificity to $30 \%$.

Although we had a relatively large sample size, and we used a $P$ value as threshold for selection in the model of 0.10 , other factors known to be associated with the development of RDS, such as maternal diabetes, being born from a multiple pregnancy and being a male fetus were not selected in our model. Individual patient data metaanalysis, in which data from individual studies are added, might be a solution to overcome this problem of statistical power.

When the results of fetal lung maturity tests are divided in 'mature' and 'immature' results, which is done in most studies on fetal lung maturity testing, the gradual maturation of the fetal lung is not taken into account. We divided our population in three categories based on gestational age and calculated the pre-test probability for RDS based on the gestational age. Unfortunately, the gestational age group $>33$ weeks covered a wide period with different risks for RDS. However, the number of patients and the number of infants with RDS were too low to divide this population into subgroups. Another limitation of our study is that the severity of RDS could not be taken into account.

The novel approach used in this study is the introduction of prediction models, rather than the use of fixed cut-off levels for $\mathrm{LBC}$ or $\mathrm{L} / \mathrm{S}$ ratio. As a consequence, the interpretation of the LBC or L/S ratio is different at different gestational ages. As can be seen from the constructed logistic models as well as from the Figs. 1, 2 and 3, a lower gestational age results in a higher probability of RDS, and thus requires a relatively high $\mathrm{LBC}$ or $\mathrm{L} / \mathrm{S}$ ratio for a similar post-test probability, as compared to a similar patient undergoing the tests at a higher gestational age [5].
In studies on fetal lung maturity testing, analysis is often limited to women who delivered within $72 \mathrm{~h}$ after amniotic fluid collection. Thereafter, it is thought that the test result is not a reliable measure of the actual state of fetal lung maturity anymore. However, as the decision to accept delivery is likely to depend on the results of the tests, the number of cases with a test result indicating mature fetal lungs, will be over represented if women who delivered after $72 \mathrm{~h}$ are excluded. The bias introduced by excluding women who delivered after a certain interval after amniocentesis is called verification bias. In our previous study, only women who delivered more than 2 weeks after amniotic fluid collection appeared to have a lower L/S ratio [7]. To correct for verification bias, we calculated the probability for verification for the included women and used this probability as relative weight in the logistic model to calculate the risk for RDS $[8,9]$.

If we want to assess whether an amniocentesis to obtain information on fetal lung maturity is useful, we should consider both the prevalence of RDS as well as the additional information obtained from either the LBC or the L/S ratio. From the prediction models that were developed in this study, we can obtain the predicted prevalence of RDS-obtained without testing, the predicted prevalence of RDS-obtained after testing with the LBC and the predicted prevalence of RDS-obtained after testing with the L/S ratio. If we assume an arbitrarily chosen threshold probability of RDS, and decide to postpone delivery in case the probability of RDS is over the threshold, then we can discriminate four categories of patients: (1) patients in whom delivery is delayed in the absence of fetal lung maturity (true-positives, 'wait correctly'); (2) patients in whom delivery is delayed in the presence of fetal lung maturity (false-positives, 'wait incorrectly'); (3) patients in whom delivery is allowed in the absence of fetal lung maturity (false-negatives, 'deliver incorrectly'); and (4) patients in whom deliv- 
Table 2 Classification of patients at risk for pre-term delivery as true-positive, false-negative, false-positive, and true-negative, for gestational age $<30$ weeks, and based on three prognostic models: (a) model without fetal lung maturity testing (b) model with LBC and (c) model with L/S ratio

\begin{tabular}{lllll}
\hline & Wait correctly & Deliver incorrectly & Wait incorrectly & Deliver correctly \\
\hline (a) No test & 54 & 7 & 24 & 15 \\
(b) LBC & 56 & 5 & 22 & 17 \\
(c) L/S ratio & 58 & 3 & 17 & 21 \\
\hline
\end{tabular}

The three groups are indexed on 100 patients

Table 3 Classification of patients at risk for pre-term delivery as true-positive, false-negative, false-positive, and true-negative, for gestational age 30-33 weeks, and based on three prognostic models: (a) model without fetal lung maturity testing (b) model with LBC and (c) model with L/S ratio

\begin{tabular}{llllr}
\hline & Wait correctly & Deliver incorrectly & Wait incorrectly & Deliver correctly \\
\hline (a) No test & 32 & 1 & 58 & 9 \\
(b) LBC & 31 & 2 & 35 & 32 \\
(c) L/S ratio & 30 & 3 & 26 & 41 \\
\hline
\end{tabular}

The three groups are indexed on 100 patients

Table 4 Classification of patients at risk for pre-term delivery as true-positive, false-negative, false-positive, and true-negative, for gestational age $>33$ weeks, and based on three prognostic models: (a) model without fetal lung maturity testing (b) model with LBC and (c) model with L/S ratio

\begin{tabular}{lllll}
\hline & Wait correctly & Deliver incorrectly & Wait incorrectly & Deliver correctly \\
\hline (a) No test & 2 & 4 & 5 & 89 \\
(b) LBC & 4 & 2 & 5 & 89 \\
(c) L/S ratio & 5 & 1 & 8 & 86 \\
\hline
\end{tabular}

The three groups are indexed on 100 patients

ery is allowed in the presence of fetal lung maturity (truenegatives, 'deliver correctly').

Table 2 shows the distribution of these four categories for a gestational age $<30$ weeks, when the threshold probability at which delivery is delayed, is set at $45 \%$. The table shows a fictive cohort of 100 patients, with a prevalence of RDS of $61 \%$. Clinical management based on the LBC would imply that 5 of these 61 women would be allowed to deliver despite fetal lung immaturity, as compared to 7 without invasive testing. On the other hand, delivery in presence of fetal lung maturity, thus preventing the risk of antepartum asphyxia or even intrauterine death, would be allowed in 17 women, instead of 15 without invasive testing. Table 2 also shows that addition of the L/S ratio would decrease the number of 'incorrect' deliveries to 3 , whereas the number of correctly delivered women would increase to 21 .

Table 3 shows the situation for a gestational age between 30 and 33 weeks. For the construction of this table, the threshold probability for RDS at which delivery is delayed, is set at $15 \%$. The argument for such a lower threshold is the lower prevalence of RDS at this gestational age. As can be seen from Table 3, performance of the LBC has limited impact on the number of women who are delivered despite fetal lung immaturity, but the number of women who are delivered in the presence of fetal lung maturity increases from 9 to 32. Addition of the L/S ratio generates a small increase in women who are allowed delivery despite fetal lung immaturity, whereas the number of women who are delivered correctly increases to 41 .

Table 4 shows the situation for a gestational age over 33 weeks. Again, the threshold probability for RDS at which delivery is delayed, is set at $15 \%$. As can be seen from this table, performance of the LBC doubles the low number of women in whom delivery is postponed correctly, whereas the number of women who are allowed delivery in the presence of fetal lung maturity does not change. Addition of the L/S ratio has only limited impact on these numbers. From Table 4, it can be seen that any strategy of assessment of the fetal lung maturity, albeit with invasive testing or without invasive testing, generates more often a false positive diagnosis of fetal lung immaturity (i.e. delay of delivery in presence of fetal lung maturity) than a true positive diagnosis of fetal lung immaturity (i.e. delay of delivery in absence of fetal lung maturity). Thus, if delivery is really warranted due to a high fetal risk, one can question whether delay of delivery based on assessment of fetal lung maturity is useful. One should also take into account that 
Table 4 describes the situation for all pregnancies with a gestational age $>33$ weeks. The ratio between false-positive and true-positive diagnoses of fetal lung immaturity will increase even more strongly at a gestational age above 38 weeks, when the prevalence of fetal lung immaturity is below $1 \%$ [11]. With such a low prevalence, the ratio between women in whom delivery is delayed correctly and women in whom delivery is delayed despite fetal lung maturity will be lower than 1:20.

Recently, Karcher et al. [12] reported on the accuracy of the lamellar body count and surfactant-to-albumin ratio in relation to gestational age in the prediction of RDS. In contrast to our study, they only recruited women at a gestational age $>34$ weeks. Consequently, the prevalence of RDS in their study was only $6 \%$. This hampers comparison with our study. Moreover, the authors did not correct for verification bias, as we did in the current study.

In summary, we can state that if one wants to assess fetal lung maturity below 30 weeks the L/S ratio is the procedure of choice, and that the value of the LBC is limited in these patients. At a gestational age between 30 and 33 weeks, assessment of fetal lung maturity with the LBC is useful. As can be derived from Fig. 2, a probability of RDS below $15 \%$ virtually rules out the probability of RDS, whereas at higher probabilities additional performance of the L/S ratio is useful. At a gestational age above 33 weeks, the value of fetal lung maturity assessment is debatable due to the low prevalence of RDS.

Whether invasive testing for fetal lung maturity is useful, will depend on the effectiveness of expectant management as compared to immediate delivery in fetuses of which lung maturity tests have indicated mature lungs. While invasive fetal lungs test predicts the presence of mature lungs correctly, immediate delivery does not improve the outcome for mother and infant as compared to expectant management, then the fetal lung test might be accurate, but it will not be of value for the patient.

The value of the fetal lung maturity tests will also depend on the individual clinical situation, for example on the risk of intrauterine death in case delivery is postponed, and on the risk of deterioration of the condition of the mother in case of severe pre-eclampsia. The clinical situations will differ in women with fetal growth restriction in singleton pregnancies, in fetal growth restriction in twin pregnancies, and in women with severe pre-eclampsia. Such situations should be addressed in decision analytic models as well as in new clinical studies on the subject.

Another potential indication for invasive fetal lung testing might be that testing might prevent unnecessary or even harmful treatment with glucocorticoids. A restrictive use of these drugs may be achieved by obtaining information on the status of the fetal lung maturation. This is especially important in elective (medically indicated) preterm deliveries. In those cases, timing of delivery might depend on information of the fetal lung maturation. In such cases glucocorticoid administration may be reserved for those cases in which fetal lung testing has indicated immaturity. This strategy should be studied in decision analytic models and possibly also in randomised controlled trials.

Conflict of interest statement None of the authors have a conflict of interest.

Open Access This article is distributed under the terms of the Creative Commons Attribution Noncommercial License which permits any noncommercial use, distribution, and reproduction in any medium, provided the original author(s) and source are credited.

\section{References}

1. Gluck L, Kulovich MV (1973) Lecithin/sphinogmyelin ratios in amniotic fluid in normal and abnormal pregnancy. Am J Obstet Gynecol 15:539-546

2. Dubin SB (1998) Assessment of fetal lung maturity. Practice parameter. Am J Clin Pathol 110:723-732

3. Neerhof MG, Dohnal JC, Ashwood ER, Lee IS, Anceschi MM (2001) Lamellar body counts: a consensus on protocol. Obstet Gynecol 97:318-320. doi:10.1016/S0029-7844(00)01134-0

4. Wijnberger LDE, Huisjes AJ, Voorbij HA, Franx A, Bruinse HW, Mol BW (2001) The accuracy of lamellar body count and lecithin/ sphingomyelin ratio in the prediction of neonatal respiratory distress syndrome: a meta-analysis. BJOG 108:583-588

5. Richardson DK, Heffner LJ (2001) Fetal-lung maturity: tests mature, interpretation not. Lancet 358:684-686. doi:10.1016/S01406736(01)05848-2

6. Giedion A, Haefliger H, Dangel P (1973) Acute pulmonary X-ray changes in hyaline membrane disease treated with artificial ventilation and positive end-expiratory pressure (PEP). Pediatr Radiol 1:145-152. doi:10.1007/BF00974058

7. Wijnberger LD, de Kleine M, Voorbij HA, Arabin B, van de Leur JJ, Bruinse HW, Visser GH, Bossuyt PM, Mol BW (2003) The effect of clinical characteristics on the lecithin/sphingomyelin ratio and lamellar body count: a cross-sectional study. J Matern Fetal Neonatal Med 14:373-382. doi:10.1080/14767050412331312210

8. Begg CB, McNeil BJ (1988) Assessment of radiological tests: control of bias and other design considerations. Radiology 167:565-569

9. Hunink MG, Richardson DK, Doubilet PM, Begg CB (1990) Testing for fetal pulmonary maturity: ROC analysis involving covariates, verification bias, and combination testing. Med Decis Making 10:201-211. doi:10.1177/0272989X9001000307

10. Steyerberg EW (1996) Prognostic modeling for clinical decision making. Scientific Thesis. Erasmus University, Rotterdam, pp 1940

11. Graziosi GC, Bakker CM, Brouwers HA, Bruinse HW (1998) Elective cesarean section is preferred after the completion of a minimum of 38 weeks of pregnancy. Ned Tijdschr Geneeskd 142:2300-2303

12. Karcher R, Sykes E, Batton D, Uddin Z, Ross G, Hockman E, Shade GH Jr (2005) Gestational age-specific predicted risk of neonatal respiratory distress syndrome using lamellar body count and surfactant-to-albumin ratio in amniotic fluid. Am J Obstet Gynecol 193:1680-1684 